\title{
Diffusion-weighted magnetic resonance imaging in preoperative assessment of non-small cell lung cancer
}

\author{
Hiroaki Nomori, MD, ${ }^{\mathrm{a}}$ Yue Cong, $\mathrm{MD},{ }^{\mathrm{a}}$ Masaru Abe, MD, ${ }^{\mathrm{a}}$ Hiroshi Sugimura, MD, ${ }^{\mathrm{a}}$ and Yoshiaki Kato, $\mathrm{RT}^{\mathrm{b}}$
}

Background: Diffusion-weighted magnetic resonance imaging (DWI) frequently shows heterogeneity of signal intensity (SI) in non-small cell lung cancer (NSCLC). The purpose of our study was to examine the association of SI and DWI patterns with histology, tumor invasiveness, lymph node metastasis, and ${ }^{18} \mathrm{~F}$-fluorodeoxyglucose positron emission tomography (FDG-PET) uptake in NSCLC.

\begin{abstract}
Methods: One hundred forty-five patients with NSCLC underwent preoperative DWI and FDG-PET. DWI patterns were visually classified as homogenous $(\mathrm{HOM})(\mathrm{n}=81)$ or heterogeneous (HET) $(n=64)$. The former was further classified as faint (faint-HOM) $(n=27)$ or dark (dark-HOM) $(n=54)$ according to a cutoff value of SI. Associations of SI and DWI patterns with tumor histology, lymphatic or vascular invasion, pleural invasion, lymph node metastasis, and FDG uptake were evaluated.

Results: All faint-HOM tumors were well-differentiated adenocarcinoma, whereas dark-HOM and HET tumors were less-differentiated adenocarcinoma or nonadenocarcinoma. Although the dark areas in HET tumors showed a dense aggregation of tumor cells, their faint areas showed abundant fibrovascular stroma or necrosis, or a well-differentiated part of adenocarcinoma. Tumor size and the frequencies of lymphatic or vascular invasion, pleural invasion, and nodal metastasis were highest in HET tumors, followed by dark-HOM and faint-HOM $(P=.1-P<.001)$ tumors. Sixty-five tumors having at least 1 of the invasions or metastasis showed significantly higher SI than the 81 tumors without $(P<.001)$. HET tumors had the highest FDG uptake, followed by dark-HOM and faint-HOM tumors; differences between the groups were significant $(P<.01$ to $P<.001)$.
\end{abstract}

Conclusions: The SI and heterogeneity of DWI reflect the histologic heterogeneity, tumor aggressiveness, and FDG-PET uptake in NSCLC. (J Thorac Cardiovasc Surg 2015;149:991-6)

See related commentary on page 997.

Although positron emission tomography (PET) using ${ }^{18} \mathrm{~F}$-fluorodeoxyglucose (FDG) has been used for TNM staging and evaluating tumor aggressiveness of non-small cell lung cancer (NSCLC), it is not a popular device all around the world because of its high cost. In contrast, magnetic resonance imaging (MRI) is popular because of not only lower cost, but also applicability for various diseases, including those that are benign. Recent diffusion-weighted magnetic resonance imaging (DWI) technology has enabled imaging of various types of malignant tumors similar to FDG-PET, including breast tumors, ${ }^{1}$ musculoskeletal tumors, ${ }^{2}$ prostate cancer, ${ }^{3}$ rectal cancer, ${ }^{4,5}$ and lung cancer. ${ }^{6}$ The mechanism of action of imaging malignant tumors by DWI is that the

\footnotetext{
From the Departments of Thoracic Surgery ${ }^{\mathrm{a}}$ and Diagnostic Radiology, ${ }^{\mathrm{b}}$ Kameda Medical Center, Chiba, Japan.

Disclosures: Authors have nothing to disclose with regard to commercial support.

Received for publication Sept 21, 2014; revisions received Dec 30, 2014; accepted for publication Jan 10, 2015; available ahead of print Feb 14, 2015.

Address for reprints: Hiroaki Nomori, MD, Department of General Thoracic Surgery,

Kameda Medical Center, 929 Higashi-cho, Kamogawa City, 296-8602 Chiba,

Japan (E-mail: hnomori@qk9.so-net.ne.jp).

$0022-5223 / \$ 36.00$

Copyright (C) 2015 by The American Association for Thoracic Surgery

http://dx.doi.org/10.1016/j.jtcvs.2015.01.019
}

diffusion of water molecules is affected by cellularity and nuclear/cytoplasmic ratio, which are different between malignant tumors and benign tissue. ${ }^{7-9}$

Although we have used DWI to image NSCLC since $2006,{ }^{10-12}$ we frequently see heterogeneity on the imaging. From the theory of DWI, its heterogeneity may reflect histologic heterogeneity within tumors, such as cellularity and nuclear/cytoplasmic ratio, which are strongly associated with tumor aggressiveness. On the other hand, conventional imaging modalities such as computed tomography (CT), FDG-PET, and T1- and T2-weighted MRI can hardly image the histologic heterogeneity of NSCLC. On the basis of our hypothesis that DWI can image the histologic heterogeneity of NSCLC, we examined the signal intensity (SI) and heterogeneity of DWI in patients with NSCLC, which were compared with histology, lymphatic or vascular invasion, pleural invasion, lymph node metastasis, and FDG uptake on PET in surgically resected specimens.

\section{MATERIALS AND METHODS Eligibility}

The study protocol for examining DWI in patients with NSCLC before surgery was approved by the Kameda Medical Center Ethics Committee Informed consent was obtained from all patients after detailed information was given by their surgeons. 


$$
\begin{aligned}
& \text { Abbreviations and Acronyms } \\
& \begin{aligned}
\mathrm{CR} & =\text { contrast ratio } \\
\mathrm{CT} & =\text { computed tomography } \\
\mathrm{DWI} & =\text { diffusion-weighted magnetic resonance } \\
& \text { imaging } \\
\text { FDG } & { }^{18} \text { F-fluorodeoxyglucose } \\
\mathrm{HET} & =\text { heterogeneous } \\
\mathrm{HOM} & =\text { homogenous } \\
\text { NSCLC } & =\text { non-small cell lung cancer } \\
\text { MRI } & =\text { magnetic resonance imaging } \\
\text { PET } & =\text { positron emission tomography } \\
\text { ROI } & =\text { region of interest } \\
\text { SI } & \text { signal intensity } \\
\text { SUV } & =\text { standardized uptake volume }
\end{aligned}
\end{aligned}
$$

\section{Patients}

Between October 2012 and December 2014, 174 patients with NSCLC underwent DWI and FDG-PET followed by surgical treatment. Of these, 11 tumors $<1 \mathrm{~cm}$ in size and 18 tumors with pure ground-glass opacity appearance were excluded. As a result, 145 tumors in 143 patients were enrolled in the study. Table 1 shows the tumor characteristics, including tumor histologic types, lymphatic or vascular invasion, pleural invasion, and lymph node metastasis. Of the 145 tumors, 63 had at least 1 of lymphatic, vascular, or pleural involvement, and lymph node metastasis, whereas 82 had none of those.

Twenty benign nodules in 20 patients who underwent DWI during the same period were used for determining the cutoff value of SI between benign and malignant nodules, which was finally used for determining the cutoff value between tumors producing faint and dark contrasts on DWI.

\section{DWI}

All MRI images were obtained with a 1.5-T system (MAGNETOM Avanto; Siemens, Munich, Germany). Conventional MRI images and DWI were acquired during the same session. The former consisted of a coronal T1-weighted sequence (repetition time/echo time $=177 \mathrm{~ms} / 4.7 \mathrm{~ms}$ ) and coronal and axial single shot spin echo T2-weighted sequences $(1000 \mathrm{~ms} / 102 \mathrm{~ms})$. The T1- and T2-weighted images were acquired at a section thickness of $5 \mathrm{~mm}$ with a $0 \mathrm{~mm}$ gap, with a $320 \times 156$ and $256 \times 194$ matrix, respectively, and a $263 \times 350 \mathrm{~mm}$ and $280 \times 350 \mathrm{~mm}$ field of view, respectively. DWI was performed in the axial plane using a spin-echo, echo-planar imaging sequence with pacing to each breath by using the following parameters: repetition time/echo time $=3100 \mathrm{~ms} / 73 \mathrm{~ms}$ with each breath, diffusion gradient encoding in 3 orthogonal directions: $\mathrm{b}=0-800 \mathrm{~s} / \mathrm{mm}^{2}$, field of view $=310 \times 350 \mathrm{~mm}, 118 \times 118$ matrix, and section thickness $=5 \mathrm{~mm}$ with a $0 \mathrm{~mm}$ gap. Fusion images were made by T2-weighted imaging and DWI for confirmation of the lesions.

\section{Measurement of SI on DWI}

The SI was measured using the region of interest (ROI) on the lesion by using Virtual Space software (AZE Co, Tokyo, Japan). The ROI was drawn around the tumor. The maximum SI within the ROI was measured for each tumor. To eliminate the fluctuations of SI caused by imaging conditions in each lesion, the contrast ratio of SI (SI-CR) was calculated by maximum SI of the lesion/mean SI of spinal cord at the same level of the lesion.

\section{Classification of DWI Patterns}

Based on visual assessment, DWI patterns were classified into homogenous $(\mathrm{HOM})(\mathrm{n}=81)$ and heterogeneous $(\mathrm{HET})(\mathrm{n}=64)$
(Figures 1-3). The former was further classified into faint (faint-HOM) $(\mathrm{n}=27)$ and dark (dark-HOM) $(\mathrm{n}=54)$ based on the cutoff value of SI-CR, as determined on the receiver operating characteristic curve to discriminate between the 20 benign and 109 malignant nodules $<3 \mathrm{~cm}$ in size; that is, 0.45 .

\section{PET-CT Scanning}

PET scanning was conducted by using PET/CT (Discovery ST; GE Medical Systems, Amersham, UK). The dose of FDG was 185 to 230 $\mathrm{MBq}$, which was determined according to body mass index. The acquisition time for PET was 2.5 or 3 minutes per table position.

\section{PET Data Analysis}

PET data were evaluated by a contrast ratio of standardized uptake value (SUV-CR) as described previously. ${ }^{13,14}$ Briefly, the SUV-CR was calculated by the equation maximum SUV of the lesion/maximum SUV of the contralateral lung.

\section{Comparison Between DWI and Histologic Findings}

Table 2 shows the relationship of DWI pattern with tumor histologic type. Although all of the 27 faint-HOM tumors were well-differentiated adenocarcinoma, the dark-HOM and HET tumors were less-differentiated adenocarcinoma or nonadenocarcinomas. To compare histologic findings between the faint and dark areas of HET tumors histologically, the materials resected by surgery were sliced on the axial plane to correspond to the plane of the DWI images. The maximum cut surface of resected tumors was compared with its axial view on DWI. The dark and faint areas were marked on sections stained with hematoxylin and eosin.

\section{Pathologic Analysis}

Hematoxylin and eosin and Elastica-van Gieson staining were performed in all sections to investigate pleural and intratumoral lymphatic

TABLE 1. Tumor characteristics

\begin{tabular}{lc}
\multicolumn{1}{c}{ Characteristic } & Value \\
\hline Tumor size (cm) & $2.5 \pm 1.5$ \\
Mean \pm standard deviation & $1.0-7.5$ \\
Range & \\
Histologic type & 114 \\
$\quad$ Adenocarcinoma & 24 \\
Squamous cell carcinoma & 4 \\
Large cell carcinoma & 2 \\
Adenosquamous cell carcinoma & 1 \\
Large cell neuroendocrine tumor & \\
Lymphatic or vascular invasion & 98 \\
- & 47 \\
+ & \\
Pleural invasion & 118 \\
P0 & 27 \\
P1-P3 & \\
Pathologic N stage & 125 \\
N0 & 20 \\
N1-N2 & \\
Lymphatic, vascular, or pleural invasion or nodal metastasis & 82 \\
- & 63 \\
+ & 145 \\
Total &
\end{tabular}

Values are presented as $\mathrm{n}$ unless otherwise noted. 


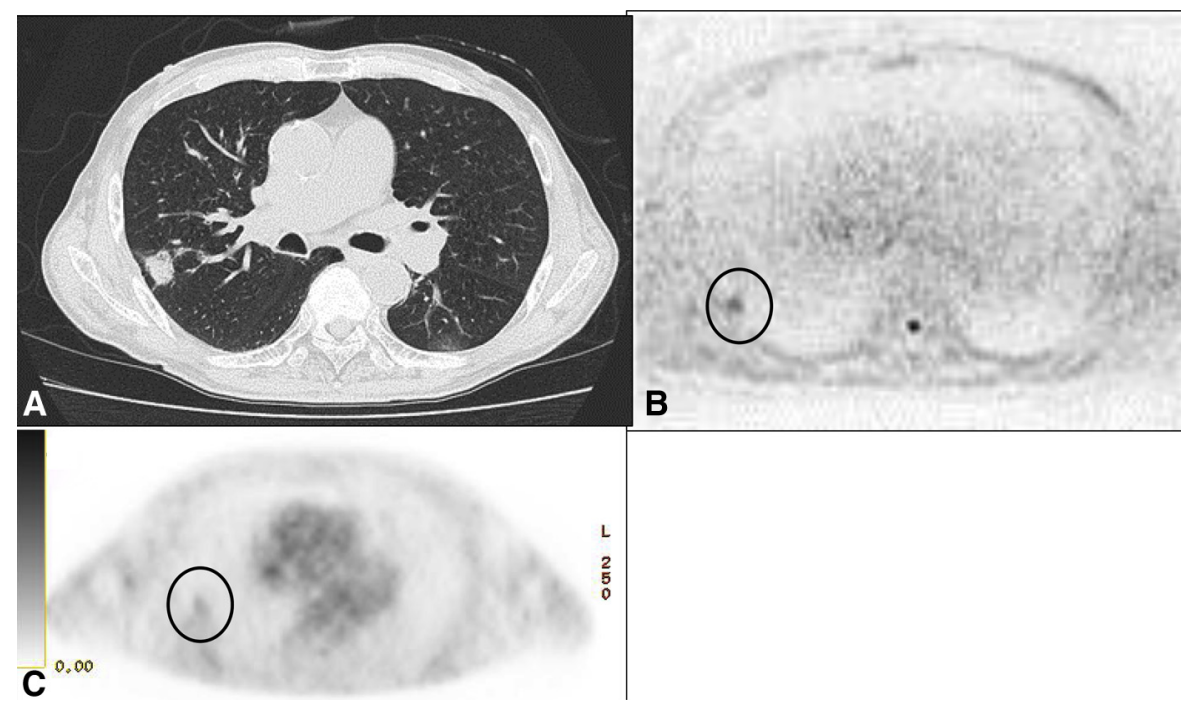

FIGURE 1. Pulmonary adenocarcinoma with faint-homogenous pattern on diffusion-weighted magnetic resonance imaging. A, Computed tomography image. B, Diffusion-weighted image. The tumor is indicated in a circle. The maximum signal intensity of the tumor is 15 and the mean signal intensity of the spinal cord is 65 ; that is, 0.23 of signal intensity contrast ratio. C, Fluorodeoxyglucose-positron emission tomography. The tumor is indicated in a circle. The maximum standardized uptake values of the tumor and contralateral lung are 1.2 and 0.52 , respectively; that is, 2.3 of the standardized uptake value contrast ratio.

or vascular invasions. Pleural invasion was classified as $\mathrm{P} 0$ to $\mathrm{P} 3$ according to International Association for the Study of Lung Cancer staging. ${ }^{15}$

\section{Statistical Analysis}

The differences in frequencies of lymphatic or vascular invasion, pleural invasion, and lymph node metastasis between the groups were examined by $\chi^{2}$ test. The differences in tumor size, SI-CR, and SUV-CR between the groups were analyzed by Mann-Whitney $U$ test.
StateMate III software (ATOMS Ltd, Tokyo, Japan) was used for statistical analyses. All values in the text and tables are given as mean \pm standard deviation.

\section{RESULTS}

The mean values of SI-CR in the faint and dark areas in the 64 HET tumors were $0.84 \pm 0.57$ and $1.72 \pm 1.01$,
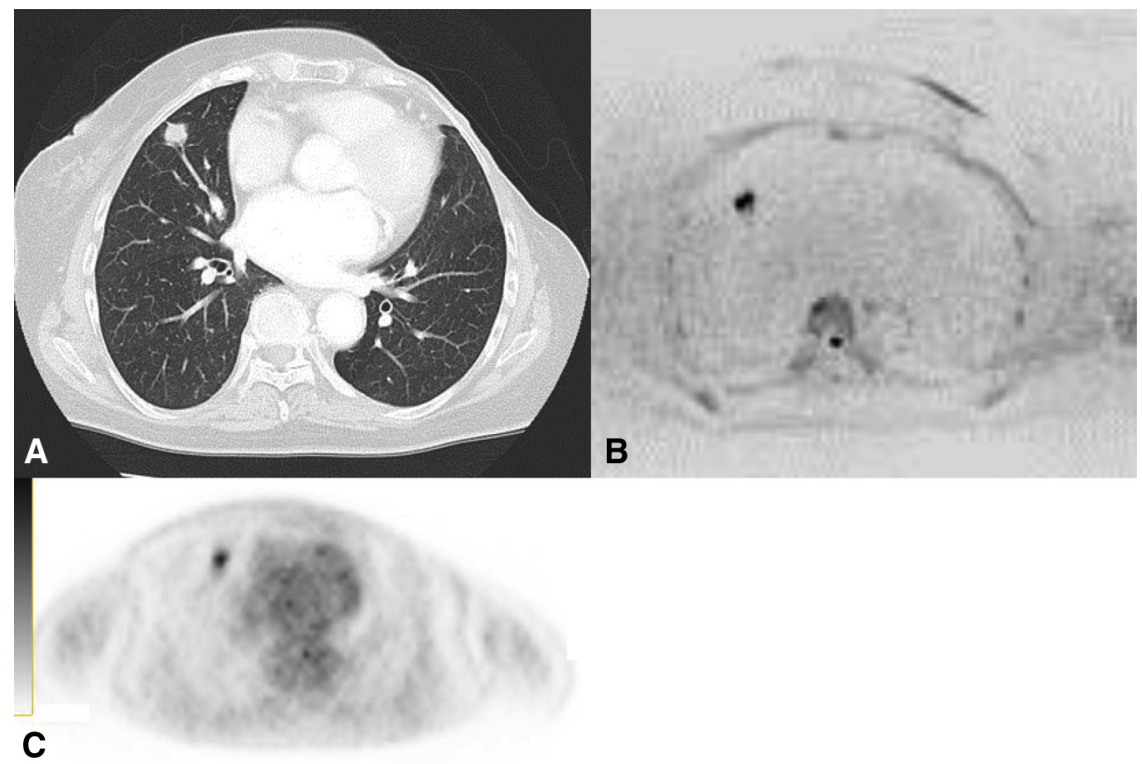

FIGURE 2. Pulmonary adenocarcinoma with dark-homogenous pattern on diffusion-weighted magnetic resonance imaging. A, Computed tomography image. B, Diffusion-weighted image. The maximum signal intensity of the tumor is 94 and the mean signal intensity of the spinal cord is 62 ; that is, 1.52 of signal intensity contrast ratio. C, Fluorodeoxyglucose-positron emission tomography. The maximum standardized uptake values of tumor and contralateral lung are 4.3 and 0.67 , respectively; that is, 6.3 of the standardized uptake value contrast ratio. 

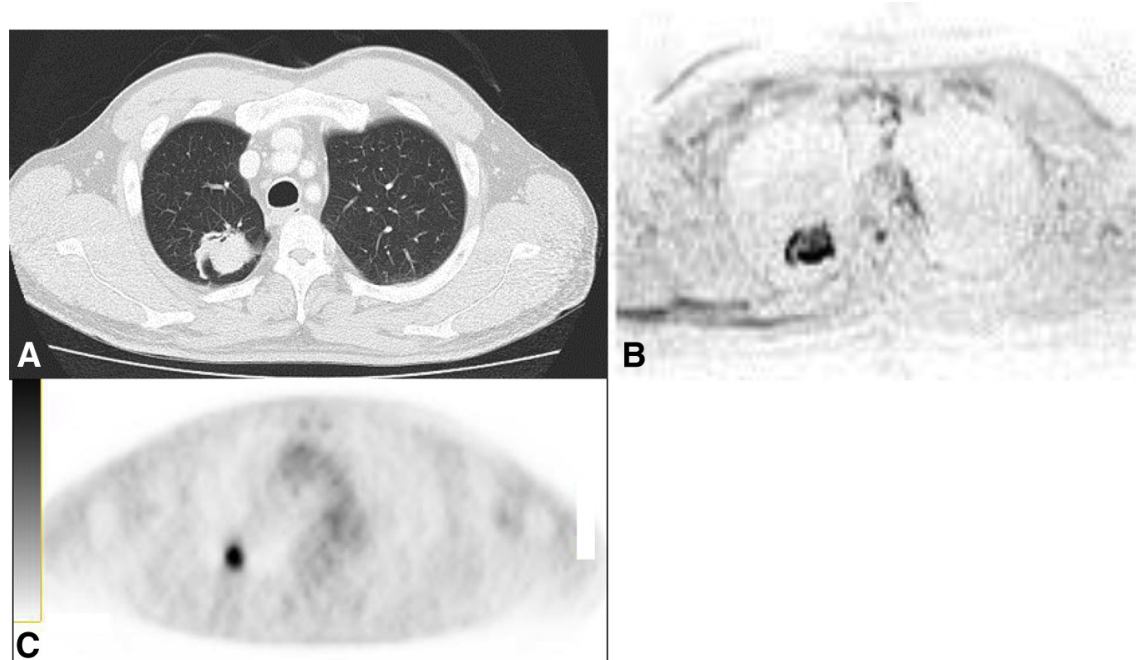

FIGURE 3. Pulmonary adenocarcinoma with heterogeneous pattern on diffusion-weighted magnetic resonance imaging. A, computed tomography image. B, Diffusion-weighted image. The maximum signal intensity of the tumor is 73 and the mean signal intensity of the spinal cord is 58 ; that is, 1.26 of signal intensity contrast ratio. The signal intensity contrast ratio of the dark area and faint area were 1.26 and 0.69 , respectively. C, Fluorodeoxyglucose-positron emission tomography. The maximum standardized uptake values of tumor and contralateral lung are 5.8 and 0.52 , respectively; that is, 11.2 of the standardized uptake ratio contrast ratio.

respectively. Although the dark areas usually showed a dense aggregation of tumor cells, the faint areas showed abundant fibrovascular stroma in 44 tumors $(69 \%)$, necrosis in 11 tumors $(17 \%)$, and a well-differentiated part of adenocarcinoma in 9 tumors $(14 \%)$.

Table 3 shows the relationship of DWI pattern with tumor size, lymphatic or vascular invasion, pleural invasion, and pathologic $\mathrm{N}$ stage. The mean tumor size was $1.7 \pm 0.6$ $\mathrm{cm}, 2.3 \pm 0.7$, and $3.0 \pm 1.3 \mathrm{~cm}$ for faint-HOM, dark-HOM, and HET tumors, respectively. The frequencies of lymphatic or vascular invasion were $0 \%, 22 \%$, and $55 \%$ for faint-HOM, dark-HOM, and HET tumors, respectively. The frequencies of pleural invasion were $0 \%, 15 \%$, and $30 \%$ for those tumors, respectively. The frequencies of lymph node metastasis were $0 \%, 9 \%$, and $23 \%$ for those, respectively. HET tumors were the largest and had the highest frequency of lymphatic or vascular invasion, pleural

TABLE 2. Correlation between DWI patterns and tumor histologic type

\begin{tabular}{lcccr}
\hline & \multicolumn{3}{c}{ DWI pattern } & \\
\cline { 2 - 4 } \multicolumn{1}{c}{ Homogenous } & & \\
\cline { 2 - 4 } \multicolumn{1}{c}{ Histologic type } & Faint & Dark & Heterogeneous & Total \\
\hline Adenocarcinoma & 27 & 44 & 44 & 115 \\
Squamous cell carcinoma & 0 & 8 & 15 & 23 \\
Large cell carcinoma & 0 & 0 & 4 & 4 \\
Adenosquamous carcinoma & 0 & 1 & 1 & 2 \\
Large cell neuroendocrine & 0 & 1 & 0 & 1 \\
$\quad$ carcinoma & & & & \\
Total & 27 & 54 & 64 & 145 \\
\hline$D W I$, Diffar
\end{tabular}

DWI, Diffusion-weighted imagnetic resonance imaging. invasion, and lymph node metastasis, followed by dark-HOM and faint-HOM $(P=.1$ to $P<.001)$.

Figure 4 shows the SI-CR of 81 tumors without lymphatic, vascular, or pleural invasion, or lymph node metastasis and 65 having at least 1 of them. The mean values of SI-CR were $0.81 \pm 0.61$ in the former and $1.81 \pm 0.94$ in the latter. The difference was significant $(P<.001)$.

Figure 5 shows the distribution of SI-CR of faint-HOM, dark-HOM, and HET tumors, of which mean values were

TABLE 3. Relationship of DWI patterns with tumor size, tumor invasiveness, and $\mathrm{pN}$ stage

\begin{tabular}{|c|c|c|c|c|c|c|}
\hline \multirow[b]{2}{*}{ Variable } & \multicolumn{3}{|c|}{ DWI pattern } & \multicolumn{3}{|c|}{$P$ value } \\
\hline & Faint & Dark & HET & $\begin{array}{c}\text { Faint } \\
\text { vs } \\
\text { dark }\end{array}$ & $\begin{array}{c}\text { Dark } \\
\text { vs } \\
\text { HET }\end{array}$ & $\begin{array}{c}\text { Faint } \\
\text { vs } \\
\text { HET } \\
\end{array}$ \\
\hline $\begin{array}{c}\text { Mean tumor } \\
\text { size }(\mathrm{cm})\end{array}$ & $1.7 \pm 0.6$ & $2.3 \pm 0.7$ & $3.0 \pm 1.3$ & .005 & $<.001$ & $<.001$ \\
\hline \multicolumn{7}{|l|}{$\begin{array}{r}\text { Lymphatic or } \\
\text { vascular } \\
\text { invasion }\end{array}$} \\
\hline- & 27 & 42 & 29 & .008 & $<.001$ & $<.001$ \\
\hline+ & 0 & 12 & 35 & & & \\
\hline \multicolumn{7}{|c|}{ Pleural invasion } \\
\hline $\mathrm{P} 0$ & 27 & 46 & 45 & .04 & .06 & .002 \\
\hline P1-P3 & 0 & 8 & 19 & & & \\
\hline \multicolumn{7}{|l|}{ pN stage } \\
\hline N0 & 27 & 49 & 49 & .1 & .04 & .006 \\
\hline N1-N2 & 0 & 5 & 15 & & & \\
\hline Total & 27 & 54 & 64 & & & \\
\hline
\end{tabular}




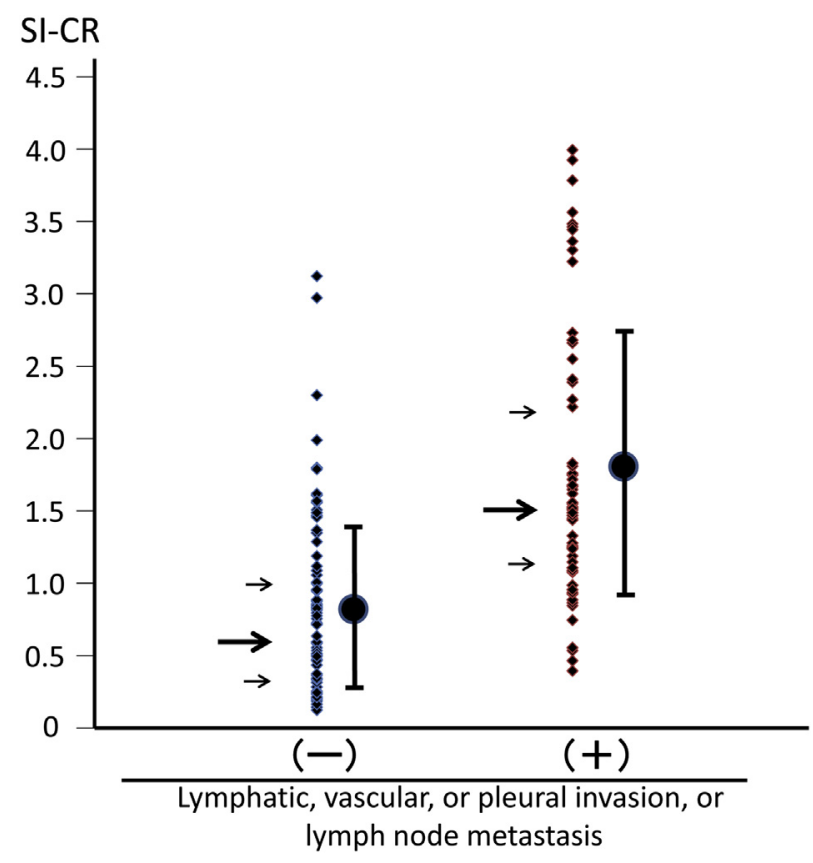

FIGURE 4. The distributions of signal intensity contrast ratio (SI-CR) in tumors with lymphatic, vascular, or pleural invasion, or lymph node metastasis and those without. The 25th percentile, median, and 75th percentile values of the former were 1.1, 1.5, and 2.23, respectively, whereas those of the latter were $0.36,0.61$, and 1.02 , respectively. A central large arrow in each distribution shows median value, and the top and bottom small arrows show 75 th and 25 th percentile values, respectively.

$0.29 \pm 0.09,1.1 \pm 0.6$, and $1.8 \pm 0.9$, respectively, showing significant differences between the groups $(P<.001)$.

Figure 6 shows the distribution of SUV-CR on FDG-PET of faint-HOM, dark-HOM, and HET tumors, of which mean values were $2.5 \pm 1.5,6.0 \pm 5.3$, and $12.8 \pm 11.5$, respectively, showing significant differences between faint-HOM and dark-HOM tumors $(P=.002)$, between dark-HOM and HET tumors $(P<.001)$, and between faint-HOM and HET tumors $(P<.001)$.

\section{DISCUSSION}

Our study highlights that heterogeneity on DWI reflects histologic heterogeneity; that is, although the dense areas in HET tumors showed a dense aggregation of tumor cells, their faint areas showed abundant fibrovascular stroma or necrosis, or a differentiated part of adenocarcinomas. HET tumors were the largest, followed by dark-HOM and faint-HOM. Also, the high SI value and heterogeneity on DWI were associated with tumor aggressiveness such as lymphatic or vascular invasion, pleural invasion, lymph node metastasis, and high SUV on FDG-PET.

It is well known that the larger a NSCLC tumor grows, the more it comes to have histologic heterogeneity. Our study showed that the HET tumors were larger than HOM tumors, and also showed that the faint areas in HET tumors showed fibrovascular stroma or necrosis—or differentiated

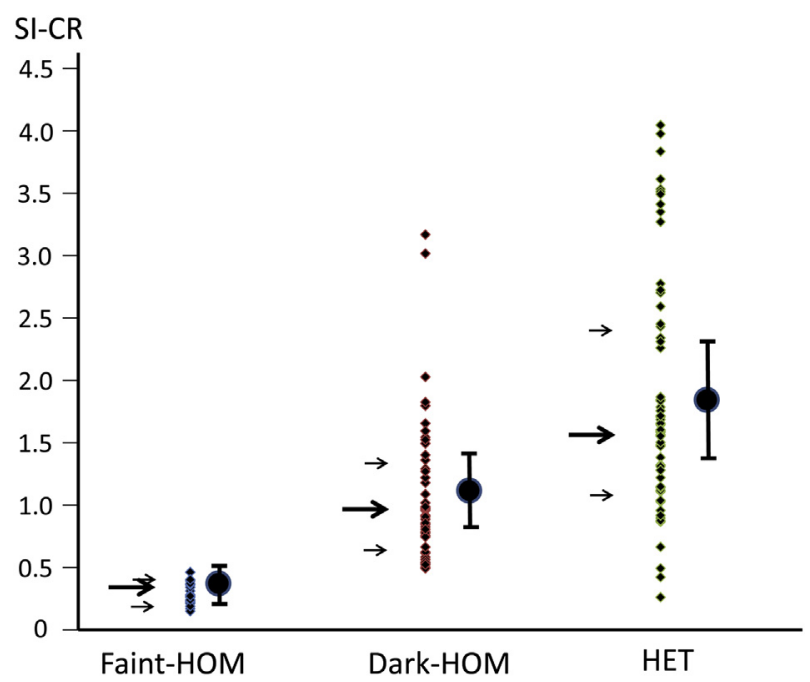

FIGURE 5. The distributions of signal intensity contrast ratio $(S I-C R)$ on diffusion-weighted magnetic resonance imaging in faint-homogenous (Faint-HOM), dark-homogenous (Dark-HOM), and heterogeneous (HET) tumors. The 25 th percentile, median, and 75 th percentile values of the faint-HOM tumors were $0.22,0.27$, and 0.36 , respectively. Images that appeared dark-HOM were $0.61,0.95$, and 1.34 , respectively, and those that were HET were 1.1, 1.58, and 2.42, respectively. A central large arrow in each distribution shows median value, and the top and bottom small arrows show 75 th and 25 th percentile values, respectively.

areas in adenocarcinomas-that had less cell-density than the dark areas. This finding is in agreement with the theory of DWI because with decreasing cell density of malignant tumors due to fibrovascular stroma or necrosis, the

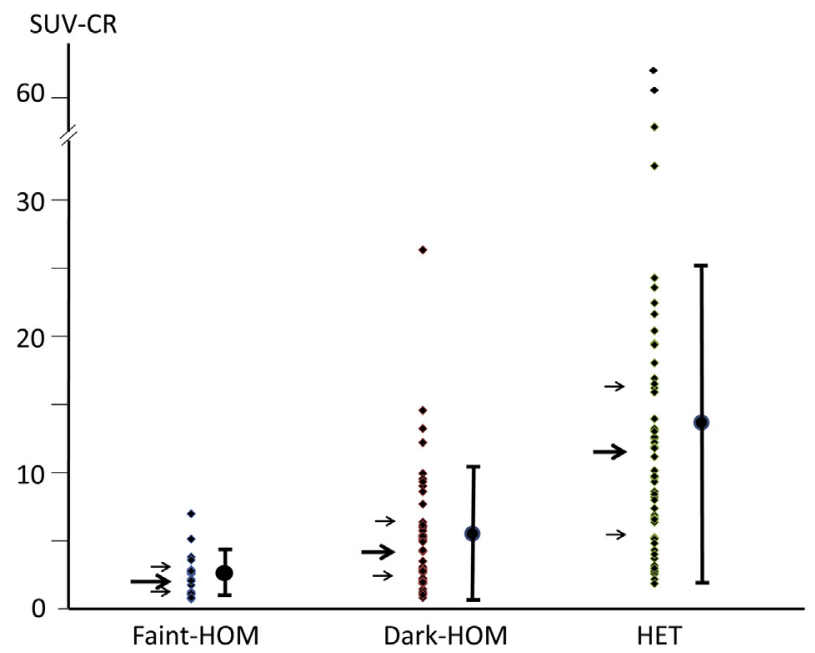

FIGURE 6. The distributions of ${ }^{18} \mathrm{~F}$-fluorodeoxyglucose positron emission tomography uptake in faint-homogenous (Faint-HOM), darkhomogenous (Dark-HOM), and heterogeneous (HET) tumors. The 25th percentile, median, and 75th percentile values of the faint-HOM tumors were $1.4,2.3$, and 3.0 , respectively. Those of dark-HOM tumors were 2.2, 4.4, and 6.5, respectively. HET tumors were 5.3, 11.2, and 16.4, respectively. A central large arrow in each distribution shows median value, and the top and bottom small arrows show 75th and 25th percentile values. 
extracellular space is increased, which increases the diffusion of water molecules, resulting in low SI. These histologic heterogeneities are usually hard to see by conventional imaging such as CT, PET, and T1- and T2-weighted MRI. It can be stated that DWI is superior to those for imaging histologic heterogeneities in NSCLC.

We showed that all of the faint-HOM tumors were differentiated adenocarcinoma without lymphatic or vascular invasion, pleural invasion, or lymph node metastasis, whereas the dark-HOM and HET tumors had more tumor aggressiveness and higher SUV on FDG-PET. These findings could be caused by the fact that the tumor cells in differentiated adenocarcinoma showing faint-HOM often have less nuclear/cytoplasmic ratio and more extracellular space than those of aggressive tumors with dark-HOM or HET. On the other hand, Ohba and colleagues ${ }^{11}$ reported that DWI could not predict the tumor invasiveness of NSCLC as well as FDG-PET, but they only measured the apparent diffusion coefficient value not evaluated the DWI pattern. Our data showed that both the SI and DWI pattern could predict the tumor aggressiveness like FDG-PET.

Limitations of the study are that we did not examine the postoperative prognosis with relation to the SI value and DWI pattern. It is well known that FDG-PET uptake can be a prognostic factor in patients with NSCLC. ${ }^{16-18}$ In the future, we will examine the postoperative prognosis with SI value and DWI pattern in patients with NSCLC.

MRI has the following advantages over PET: patients do not have to fast before examination, there is no radiation exposure, less time is required for the examination (30 minutes in DWI vs 90 minutes in PET-CT), and the cost is considerably less ( $\$ 100$ for DWI vs $\$ 700$ for PET-CT in Japan). On the other hand, MRI is inferior to PET for whole body imaging.

\section{CONCLUSIONS}

The SI and heterogeneity of NSCLC on DWI could be useful for predicting not only histologic heterogeneity, but also tumor aggressiveness, which may help guide treatment decisions.

\section{References}

1. Woodhams R, Matsunaga K, Kan S, Hata H, Ozaki M, Iwabuchi K, et al. ADC mapping of benign and malignant breast tumors. Magn Reson Med Sci. 2005;4: $35-42$.
2. Hayashida Y, Yakushiji T, Awai K, Katahira K, Nakayama Y, Shimomura O, et al. Monitoring therapeutic responses of primary bone tumors by diffusion-weighted image: initial results. Eur Radiol. 2006;16:2637-43.

3. Reinsberg SA, Payne GS, Riches SF, Ashley S, Brewster JM, Morgan VA, et al. Combined use of diffusion-weighted MRI and 1H MR spectroscopy to increase accuracy in prostate cancer detection. AJR Am J Roentgenol. 2007; 188:91-8.

4. Nasu K, Kuroki Y, Kuroki S, Murakami K, Nawano S, Moriyama N. Diffusion-weighted single shot echo planar imaging of colorectal cancer using a sensitivity-encoding technique. Jpn J Clin Oncol. 2004;34:620-6.

5. Dzik-Jurasz A, Domenig C, George M, Wolber J, Padhani A, Brown G, et al. Diffusion MRI for prediction of response of rectal cancer to chemoradiation. Lancet. 2002;360:307-8.

6. Matoba M, Tonami H, Kondou T, Yokota H, Higashi K, Toga H, et al. Lung carcinoma: diffusion weighted MR imaging - preliminary evaluation with apparent diffusion coefficient. Radiology. 2007;243:570-7.

7. Wang J, Takashima S, Takayama F, Kawakami S, Saito A, Matsushita T, et al. Head and neck lesions: characterization with diffusion-weighted echo-planar MR imaging. Radiology. 2001;220:621-30.

8. Sumi M, Takagi Y, Uetani M, Morikawa M, Hayashi K, Kabasawa H, et al. Diffusion-weighted echoplanar MR imaging of salivary glands. AJR Am J Roentgenol. 2002;178:959-65

9. Takahara T, Imai Y, Yamashita T, Yasuda S, Nasu S, Van Cauteren M. Diffusion weighted whole body imaging with background body signal suppression (DWIBS): technical improvement using free breathing, STIR and high resolution 3D display. Radiat Med. 2004;22:275-82.

10. Mori T, Nomori H, Ikeda K, Kawanaka K, Shiraishi S, Katahira K, et al. Diffusion-weighted magnetic resonance imaging for diagnosing malignant pulmonary nodules/masses: comparison with positron emission tomography. J Thorac Oncol. 2008;3:358-64.

11. Ohba Y, Nomori H, Mori T, Ikeda K, Shibata H, Kobayashi H, et al. Is Diffusionweighted magnetic resonance imaging superior to fluorodeoxyglucose-positron emission tomography in non-small cell lung cancer? J Thorac Cardiovasc Surg. 2009;138:439-45.

12. Nomori H, Mori T, Ikeda K, Kawanaka K, Shiraishi S, Katahira K, et al. Diffusion-weighted magnetic resonance imaging can be used in place of positron emission tomography for $\mathrm{N}$ staging of non-small cell lung cancer with fewer false-positive results. J Thorac Cardiovasc Surg. 2008;135:816-22.

13. Nomori H, Watanabe K, Ohtsuka T, Naruke T, Suemasu K, Uno K. Visual and semiquantitative analyses for F-18 fluorodeoxyglucose PET scanning in pulmonary nodules $1 \mathrm{~cm}$ to $3 \mathrm{~cm}$ in size. Ann Thorac Surg. 2005;79:984-8.

14. Ohba Y, Nomori H, Shibata H, Kobayashi H, Mori T, Shiraishi S, et al. Evaluation of visual and semiquantitative assessments of fluorodeoxyglucose-uptake on PET scans for the diagnosis of pulmonary malignancies 1 to $3 \mathrm{~cm}$ in size. Ann Thorac Surg. 2009;87:886-91.

15. Goldstraw P, ed. Staging manual in thoracic oncology. Orlando, FL: Rx Press; 2009:85-9.

16. Cerfolio RJ, Bryant AS, Ohja B, Bartolucci AA. The maximum standardized uptake values on positron emission tomography of a non-small cell lung cancer predict stage, recurrence, and survival. J Thorac Cardiovasc Surg. 2005;130: 151-9.

17. Vansteenkiste JF, Stroobants SG, Dupont PJ, De Leyn PR, Verbeken EK, Deneffe GJ, et al. Prognostic importance of the standardized uptake value on (18)F-fluoro-2-deoxy-glucose-positron emission tomography scan in nonsmall-cell lung cancer: an analysis of 125 cases. Leuven Lung Cancer Group. J Clin Oncol. 1999;17:3201-6.

18. Ohtsuka T, Nomori H, Watanabe K, Kaji M, Naruke T, Suemasu K, et al. Prognostic significance of $\mathrm{F}^{18}$-fluorodeoxyglucose uptake on positron emission tomography in patients with pathologic stage I lung adenocarcinoma. Cancer. 2006;107:2468-73. 